\title{
PROFISSIONAL DA INFORMAÇÃO, BIBLIOTECAS UNIVERSITÁRIAS E A PRODUÇÃO DA INFORMAÇÃO NO CONTEXTO ATUAL
}

INFORMATION PROFESSIONAL, ACDEMIC LIBRARIES AND INFORMATION PRODUCTION IN THE CURRENT CONTEXT

Gildenir Carolino Santos ${ }^{1}$, Danielle Thiago Ferreira ${ }^{2}$

${ }^{1}$ Universidade Estadual de Campinas. Sistema de Bibliotecas

2 Universidade Estadual de Campinas. Biblioteca da Área de Engenharia e Arquitetura

Correspondência

${ }^{1}$ Gildenir Carolino Santos

Universidade Estadual de Campinas

Sistema de Bibliotecas

Campinas, SP.

E-mail: rdbci@unicamp.br

ORCID: http://orcid.org/0000-0002-4375-6815

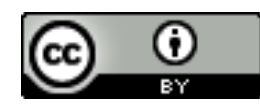

JITA: AA. Library and Information Science as a field.

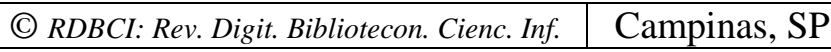

v.15

n. 1

p.282-288

maio/ago.2017 
Caros Leitores,

Estamos lançando mais um fascículo correspondente ao ano de 2017 (v.15, n.2). Desde que fizemos uso do mecanismo Ahead of Print (AOP), o crescimento de visualizações (29.876) e downloads (17.275) da revista tiveram grandes proporções nesses quatro primeiros meses de 2017, conforme apontam os indicadores estatísticos ${ }^{1}$ disponíveis na Revista. Ficamos satisfeitos, pois isso demonstra o quanto a RDBCI está sendo acessada e apreciada por seus leitores que anseiam por novidades na área e por novas pesquisas para leitura.

O benefício desse mecanismo estimulou diversos autores a procurarem a RDBCI para publicarem seus manuscritos, permitindo assim, não termos apenas autores do nosso campo de atuação, mas também autores que atuam nas mais diversas áreas do conhecimento.

A RDBCI, em sua trajetória, busca agregar os trabalhos aprovados a uma temática contemplando tanto a atuação do profissional da informação, o papel da biblioteca universitária nas diversas frentes e o contexto atual da produção de informação e seu gerenciamento. Dessa forma, a temática trazida nesse número é "Profissional da informação, bibliotecas universitárias e a produção da informação no contexto atual".

No editorial desta edição abordaremos o profissional da informação tanto bibliotecário quanto arquivista, apresentando as competências de ambos a partir da formação curricular, atuando em parceria com os profissionais da área da tecnologia da informação em relação à segurança da informação, e nas bibliotecas universitárias quanto à dramaturgia infantil.

No entanto, conforme Targino (2000, p.62), a expressão Profissional da Informação,

disseminada em nível mundial por teóricos como Madison (1990), 1995) e fortalecida pela Federação Internacional de Informação e Documentação (FID), ao criar um grupo de estudo dedicado ao moderno profissional da informação (MPI), é relativamente nova, haja vista que se vincula à chamada sociedade da informação ou sociedade do conhecimento. Esta caracteriza-se pela ênfase dada à informação e ao seu acesso à informação, ocasionando transformações profundas nos sistemas de produção e consolidando o setor quaternário da economia, que incorpora todos os indivíduos, instituições, processos, produtos e atividades que fazem parte do ciclo de vida da informação desde a sua geração - inovação científica, artística e tecnológica - até o seu consumo - absorção e aplicação. (passados 17 anos desse artigo, o termo "moderno profissional da informação" não é mais relativamente novo. Ele já se incorpora no nosso campo de conhecimento como um termo sinônimo para o bibliotecário - grifo nosso).

\footnotetext{
${ }^{1}$ Fonte: Site da RDBCI - Estatística. Disponível em: <http://periodicos.sbu.unicamp.br/ojs/index.php/ rdbci/statistics>. Acesso em: 28 abr. 2017.
}

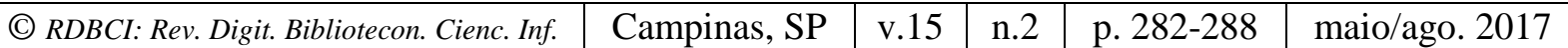


Sendo assim, daremos destaque nessa edição ao profissional da informação que atua nas bibliotecas universitárias, que se consolida, dentre os demais tipos de bibliotecas, como a que mais necessita de atualização e a que leva ao conhecimento da sociedade novos experimentos, pesquisas e tendências. Fazendo com que o profissional da informação reflita sobre sua postura, habilidades e competências, reforçando seu papel de mediador e disseminador da produção do conhecimento gerado no contex to atual.

Diante da temática apresentada, teremos nessa edição a composição de sete (7) artigos, três (3) artigos de pesquisa e uma (1) resenha.

Iniciando com a Seção Artigos, temos o manuscrito de Jorge Santa Anna (Universidade Federal do Espírito Santo) sobre "O arquivista como moderno profissional da informação: análise de competências à luz da literatura e da formação curricular". Nesse trabalho o autor analisa as competências do MIP relacionando-as ao arquivista, com base na literatura e na formação curricular e apresenta o que vem sendo publicado sobre o MIP; compara as competências do MIP com o arquivista; e investiga essas competências no âmbito da formação arquivística.

No artigo seguinte intitulado "O profissional da informação e a dramaturgia infantil: estudo sobre a produção científica em periódicos da ciência da informação entre $2000 e$ 2011 ", Clarice Fortkamp Caldin e Deyse Soares (ambas da Universidade Federal de Santa Catarina), tratam da produção científica em periódicos sobre a atuação do profissional da informação na dramaturgia infantil no período compreendido entre 2000 e 2011, tendo como objetivo investigar, por meio da produção bibliográfica na área de Ciência da Informação, o envolvimento dos bibliotecários em especial e dos profissionais da informação com a dramaturgia infantil.

Mirian Clavico Alves (Universidade Estadual de Campinas) em parceria com Leandro Innocentini Lopes de Faria e Roniberto Morato do Amaral (ambos da Universidade Federal de São Carlos), apresenta o trabalho intitulado: "Visualização de informação para simplificar o entendimento de indicadores sobre avaliação da ciência e tecnologia", que trata sobre crescimento da atividade científica e o desenvolvimento de novas tecnologias voltadas para a divulgação do conhecimento e suas contribuições para a geração de diversos indicadores, muito utilizados como instrumentos de avaliação da ciência e tecnologia. Também analisam um conjunto de indicadores científicos, a partir de nove programas de pós-graduação da área de economia avaliados pelo Sistema de Avaliação da Capes no triênio de 2013.

No quarto artigo sob o título: "Violência contra a mulher: uma relação entre dimensões subjetivas e a produção de informação", os autores Mariana de Lima Campos e Gustavo Henrique Moreira Dias Almeida (ambos da Fundação João Pinheiro), por meio de uma revisão bibliográfica e documental sobre o tema "violência contra a mulher", analisam e referem-se à inserção de dados no Sistema de Informações de Agravos de Notificação (SINAN), do Ministério da Saúde. Os autores concluem por inferência que valores arraigados

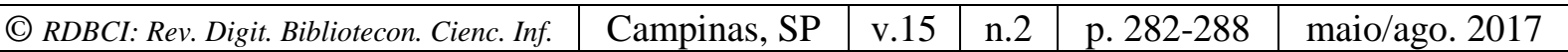


e instituídos culturalmente na sociedade refletem na informação produzida e em maior nível, na existência de subnotificações, constituindo-se como um dos aspectos negativos em relação à inserção de dados no sistema e ao enfrentamento da violência contra as mulheres.

Na sequência Zayr Claudio Gomes da Silva (Universidade Federal da Paraíba) e Edivanio Duarte de Souza (Universidade Federal de Alagoas) apresentam o texto sobre "Indicadores da produção colaborativa na arquitetura da informação". Os autores descrevem as condições da produção colaborativa na arquitetura da informação, considerando-a como subárea de estudo da Ciência a Informação. Para tanto, abordam especificamente, indicadores da produção científica que compreendem temáticas de estudo, tipologia e formação da autoria de programas de pós-graduação e áreas a que se encontram vinculadas, entre outros.

No sexto artigo Juliana Soares Lima, Ana Rafaela Sales de Araújo, Francisco Edvander Pires Santos, Luiz Gonzaga Mota Barbosa e Izabel Lima dos Santos (todos da Universidade Federal do Ceará), apresentam o artigo sobre a "Segurança da informação em bibliotecas universitárias: a atuação do bibliotecário no planejamento e na implantação de novas políticas institucionais". Os autores apresentam uma discussão sobre a atuação do bibliotecário como um profissional importante no planejamento, na elaboração e na implantação de uma política de segurança da informação em bibliotecas universitárias, trabalhando em conjunto com os profissionais da área de tecnologia da informação. $\mathrm{O}$ principal ponto nesse artigo é que os autores discorrem acerca das principais pragas virtuais existentes que tendem a infectar os computadores das bibliotecas e quais as principais tomadas de decisão referentes à segurança da informação devem ser implantadas.

No último artigo dessa seção, Orandi Mina Falsarella, Celeste Aída Sirotheau Corrêa Jannuzzi e Cibele Roberta Sugahara (todos da Pontifícia Universidade Católica de Campinas) nos brindam com o texto: "Gestão estratégica empresarial: proposição de um modelo de monitoramento informacional na era do big data". Este trabalho tem como objetivo propor um Modelo de Monitoramento Informacional que possa subsidiar a gestão estratégica empresarial, de modo a identificar ameaças, oportunidades, forças e fraquezas, propiciando a obtenção de informações importantes para a elaboração e acompanhamento do planejamento estratégico empresarial. Os autores concluem que é possível observar que um sistema de inteligência organizacional e competitiva pode ser ampliado e evoluído ao utilizar as características de big data e agente de software inteligente, resultando no modelo proposto.

Na Seção Artigo de Pesquisa damos destaque ao texto "Bibliotecas universitárias das instituições estaduais de ensino superior paranaenses e a mediação da informação no facebook" de autoria dos bibliotecários João Arlindo dos Santos Neto e Oswaldo Francisco de Almeida Júnior (ambos da Universidade Estadual Paulista). Os autores apresentam uma pesquisa sobre as bibliotecas universitárias e as formas de mediação da informação através das ferramentas web, em especial das redes sociais tendo como objetivo, identificar e analisar a mediação da informação no facebook.

\begin{tabular}{l|l|l|l|l|l}
\hline (C) RDBCI: Rev. Digit. Bibliotecon. Cienc. Inf. & Campinas, SP & v.15 & n.2 & p. 282-288 & maio/ago. 2017 \\
\hline
\end{tabular}


Na segunda pesquisa, sob o título "As percepções e práticas de publicação em acesso aberto dos pesquisadores de dois programas de pós-graduação em Engenharia”, Ariadne Chloe Furnival e Daniel André Rigo Guirra (ambos da Universidade Federal de São Carlos) apresentam os resultados de um estudo que buscou conhecer as práticas e percepções de pesquisadores credenciados em dois programas de pós-graduação em Engenharia, avaliados com nota sete pela CAPES, em relação à publicação em canais de acesso aberto comparado com os canais restritos, de assinatura.

No último texto da seção Artigos de Pesquisas, intitulado: "Proposta de aplicabilidade da preservação digital ao prontuário eletrônico do paciente", Virginia Bentes Pinto e Odete Máyra Mesquita Sales (ambas da Universidade Federal do Ceará) apresentam os resultados preliminares de uma pesquisa sobre a preservação e a curadoria digital, bem como a possibilidade de aplicabilidade ao contexto da documentação sanitária, enquanto memória da saúde. Sendo assim, o objetivo básico do artigo foi estudar a literatura referente à preservação e à curadoria digital, com ênfase no padrão OAIS, considerando a possibilidade de sua aplicação para o desenvolvimento de um repositório digital de prontuários eletrônicos de paciente, observando o ordenamento jurídico concernente a esse tipo de documento.

Em Resenhas, temos a resenha elaborada por um dos editores da RDBCI da segunda edição do livro do bibliotecário e professor da Universidade de Brasília Murilo Bastos da Cunha, intitulado "Para saber mais: fontes de informação em ciência e tecnologia", publicada única e exclusivamente no formato eletrônico e editado pela Briquet de Lemos, editora tambem especializada na área da Biblioteconomia e Ciência da Informação.

Dessa forma, encerramos o nosso editorial com a certeza de que os artigos organizados nessa edição terão destino certo, mantendo nossos leitores atualizados no que diz respeito a área da Biblioteconomia e Ciência da Informação, objetivando fomentar novas pesquisas e contribuir para a sociedade científica.

Tenham uma ótima leitura!

Gildenir Carolino Santos

Danielle Thiago Ferreira

Editores Científicos / RDBCI

Abr. 2017

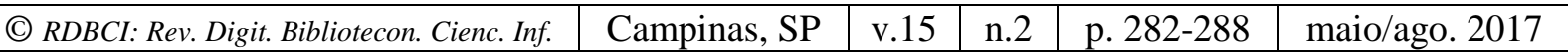




\section{REFERÊNCIAS}

ALVES, Mirian Clavico; FARIA, Leandro Innocentini Lopes de; AMARAL, Roniberto Morato do. Visualização de informação para simplificar o entendimento de indicadores sobre avaliação da ciência e tecnologia. RDBCI: Revista Digital de Biblioteconomia e Ciência da Informação, Campinas, SP, v. 15, n. 2, abr. 2017. ISSN 1678-765X. Disponível em: <http://periodicos.sbu.unicamp.br/ojs/index.php/rdbci/article/view/8646366 >. Acesso em: 28 abr. 2017. doi:http://dx.doi.org/10.20396/rdbci.v15i2.8646366.

CALDIN, Clarice Fortkamp; SOARES, Deyse. O profissional da informação e a dramaturgia infantil: estudo sobre a produção científica em periódicos da ciência da informação entre 2000 e 2011. RDBCI: Revista Digital de Biblioteconomia e Ciência da Informação, Campinas, SP, v. 15, n. 2, abr. 2017. ISSN 1678-765X. Disponível em: <http://periodicos.sbu.unicamp.br/ojs/index.php/rdbci/article/view/8645327>. Acesso em: 28 abr. 2017. doi:http://dx.doi.org/10.20396/rdbci.v15i2.8645327.

CAMPOS, Mariana de Lima; ALMEIDA, Gustavo Henrique Moreira Dias. Violência contra a mulher: uma relação entre dimensões subjetivas e a produção de informação.. RDBCI:

Revista Digital de Biblioteconomia e Ciência da Informação, Campinas, SP, v. 15, n. 2, abr. 2017. ISSN 1678-765X. Disponível em: <http://periodicos.sbu.unicamp.br/ojs/index. php/rdbci/article/view/8645969>. Acesso em: 28 abr. 2017. doi:http://dx.doi.org/10.20396/ rdbci.v15i2.8645969.

FALSARELLA, Orandi Mina; JANNUZZI, Celeste Aída Sirotheau Corrêa; SUGAHARA, Cibele Roberta. Gestão estratégica empresarial: proposição de um modelo de monitoramento informacional na era do big data. RDBCI: Revista Digital de Biblioteconomia e Ciência da Informação, Campinas, SP, v. 15, n. 2, abr. 2017. ISSN 1678-765X. Disponível em: $<$ http://periodicos.sbu.unicamp.br/ojs/index.php/rdbci/article/view/8647124>. Acesso em: 28 abr. 2017. doi:http://dx.doi.org/10.20396/rdbci.v15i2.8647124.

FURNIVAL, Ariadne Chloe; GUIRRA, Daniel Andre Rigo. As percepções e práticas de publicação em acesso aberto dos pesquisadores de dois programas de pós-graduação em engenharia. RDBCI: Revista Digital de Biblioteconomia e Ciência da Informação, Campinas, SP, v. 15, n. 2, abr. 2017. ISSN 1678-765X. Disponível em:

<http://periodicos.sbu.unicamp.br/ojs/index.php/rdbci/article/view/8646398 >. Acesso em: 28 abr. 2017. doi:http://dx.doi.org/10.20396/rdbci.v15i2.8646398.

LIMA, Juliana Soares et al. Segurança da informação em bibliotecas universitárias: a atuação do bibliotecário no planejamento e na implantação de políticas institucionais. RDBCI:

Revista Digital de Biblioteconomia e Ciência da Informação, Campinas, SP, v. 15, n. 2, abr. 2017. ISSN 1678-765X. Disponível em: <http://periodicos.sbu.unicamp.br/ojs/index. php/rdbci/article/view/8646416>. Acesso em: 28 abr. 2017. doi:http://dx.doi.org/10.20396/ rdbci.v15i2.8646416.

PINTO, Virginia Bentes; SALES, Odete Máyra Mesquita. Proposta de aplicabilidade da preservação digital ao prontuário eletrônico do paciente. RDBCI: Revista Digital de Biblioteconomia e Ciência da Informação, Campinas, SP, v. 15, n. 2, abr. 2017. ISSN

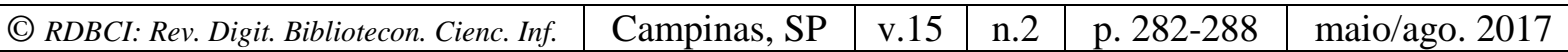


1678-765X. Disponível em: <http://periodicos.sbu.unicamp.br/ojs/index.php/rdbci/ article/view/8646311>. Acesso em: 28 abr. 2017. doi:http://dx.doi.org/10.20396/rdbci.v15i2. $\underline{8646311 .}$

SANTA ANNA, Jorge. O arquivista como moderno profissional da informação (MIP): análise de competências à luz da literatura e da formação curricular. RDBCI: Revista Digital de Biblioteconomia e Ciência da Informação, Campinas, SP, v. 15, n. 2, abr. 2017. ISSN 1678-765X. Disponível em: 〈http://periodicos.sbu.unicamp.br/ojs/index.php/rdbci/article/ view/8644523>. Acesso em: 28 abr. 2017. doi:http://dx.doi.org/10.20396/rdbci.v15i2. $\underline{8644523 .}$.

SANTOS, Gildenir Carolino. Para saber mais além...(Resenha). RDBCI: Revista Digital de Biblioteconomia e Ciência da Informação, Campinas, SP, v. 15, n. 2, abr. 2017. ISSN 1678-765X. Disponível em: 〈http://periodicos.sbu.unicamp.br/ojs/index.php/rdbci/article/ view/8648771 >. Acesso em: 28 abr. 2017. doi:http://dx.doi.org/10.20396/rdbci.v15i2. $\underline{8648771 .}$

SANTOS NETO, João Arlindo dos; ALMEIDA JÚNIOR, Oswaldo Francisco de. Bibliotecas universitárias das instituições estaduais de ensino superior paranaenses e a mediação da informação no Facebook. RDBCI: Revista Digital de Biblioteconomia e Ciência da Informação, Campinas, SP, v. 15, n. 2, abr. 2017. ISSN 1678-765X. Disponível em: 〈http://periodicos.sbu.unicamp.br/ojs/index.php/rdbci/article/view/8648210 >. Acesso em: 28 abr. 2017. doi:http://dx.doi.org/10.20396/rdbci.v15i2.8648210.

SILVA, Zayr Claudio Gomes da; SOUZA, Edivanio Duarte de. Indicadores da produção colaborativa na arquitetura da informação. RDBCI: Revista Digital de Biblioteconomia e Ciência da Informação, Campinas, SP, v. 15, n. 2, abr. 2017. ISSN 1678-765X. Disponível em: <http://periodicos.sbu.unicamp.br/ojs/index.php/rdbci/article/view/8647357>. Acesso em: 28 abr. 2017. doi:http://dx.doi.org/10.20396/rdbci.v15i2.8647357.

TARGINO, Maria das Graças. Quem é o profissional da informação? Transinformação, Campinas, v.12, n.2, p.61-69, jul./dez. 2000. Disponível em: 〈http://periodicos.puccampinas.edu.br/seer/index.php/transinfo/issue/view/246>. Acesso em: 28 abr. 2017.
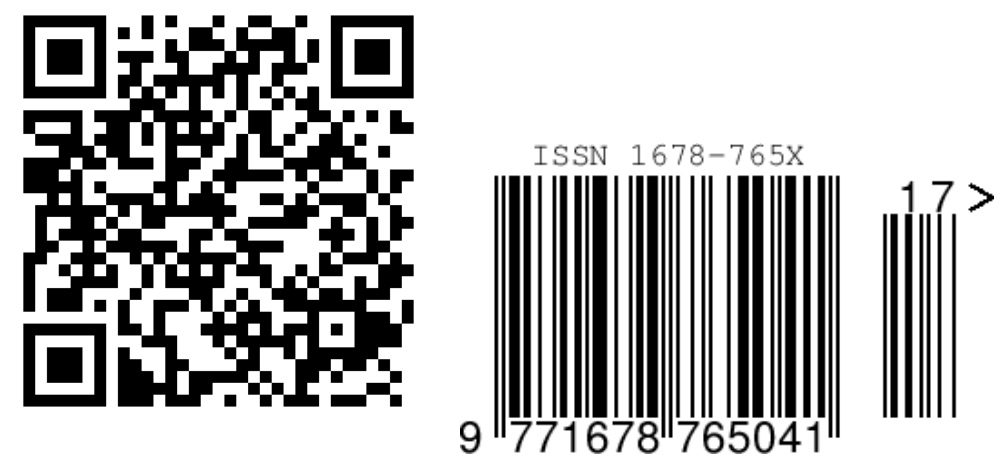

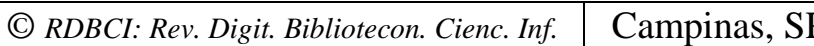

\begin{tabular}{l|l|l|l} 
v.15 & n.2 & p. $282-288$
\end{tabular}

maio/ago. 2017 